\title{
Erratum: Focus point in dark matter selected high-scale supersymmetry
}

\author{
Sibo Zheng \\ Department of Physics, Chongqing University, \\ Chongqing, 401331 P.R. China \\ E-mail: sibozheng.zju@gmail.com
}

ERRATUM TO: JHEP03(2015)098

ArXIV EPRINT: 1501.05698

There is a sign error in eq. (2.2) in the published version, which should be corrected as,

$$
\begin{aligned}
2 m_{H_{u}}^{2}(v)=I[M] & \left(m_{\tilde{Q}_{3}}^{2}[M]+m_{\tilde{U}_{3}}^{2}[M]+m_{H_{u}}^{2}[M]-A_{t}^{2}[M]\right) \\
& +I[M]^{2} A_{t}^{2}[M]-\left(m_{\tilde{Q}_{3}}^{2}[M]+m_{\tilde{U}_{3}}^{2}[M]-m_{H_{u}}^{2}[M]\right) .
\end{aligned}
$$

As a consequence, eq. (2.7) shoud be also corrected as,

$$
-x\left(I^{2}[M]-I[M]\right)+y(1-I[M])-1-I[M]=0 .
$$

Due to the sign change in eq. (2.7), figure 1, figure 2, figure 3 and figure 6 should be corrected. Details are presented below.

Here a few comments are in order, regarding the fit to the observed Higgs mass in figure 2 and figure 3. $i$ ), Given the same $m_{0}$, compare the four contours of Higgs mass in the four panels in either figure 2 or figure 3. It is shown that the focus point value of $y$ at the crossing point decreases as $M$ approaches to the GUT scale. The reason is partially due to the fact that $I[M]$ decreases as $M$ increases. $i i$ ), Given the same $M$, compare the contours of Higgs mass in each panel in either figure 2 or figure 3. As expected, the focus point value of $y(x)$ at the crossing point increases (decreases) as $m_{0}$ increases. Moreover, there are more crossing points in the case with larger $M$, In this sense the focusing may be more easily achieved in high-scale other than low-scale SUSY.

Despite the corrections above, we would like to mention that the main results and conclusions still hold as in the published version.

Open Access. This article is distributed under the terms of the Creative Commons Attribution License (CC-BY 4.0), which permits any use, distribution and reproduction in any medium, provided the original author(s) and source are credited. 


\section{$\mathrm{M}(\mathrm{GeV})$}

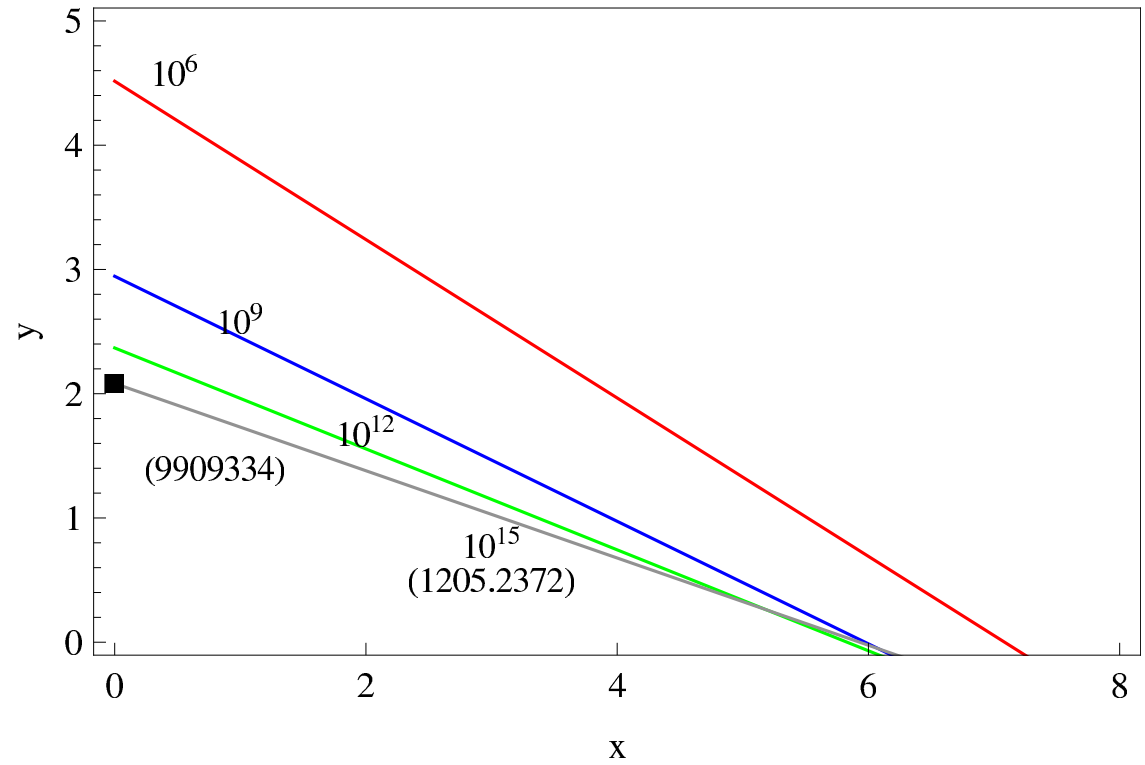

Figure 1. Focusing lines in the two-parameter plane of $(x, y)$ for $M=\left\{10^{6}, 10^{9}, 10^{12}, 10^{15}\right\} \mathrm{GeV}$. Any point in each focusing line generates the focusing phenomenon, $m_{H_{u}}^{2}[v] \simeq 0$. See the text for comments on references. 

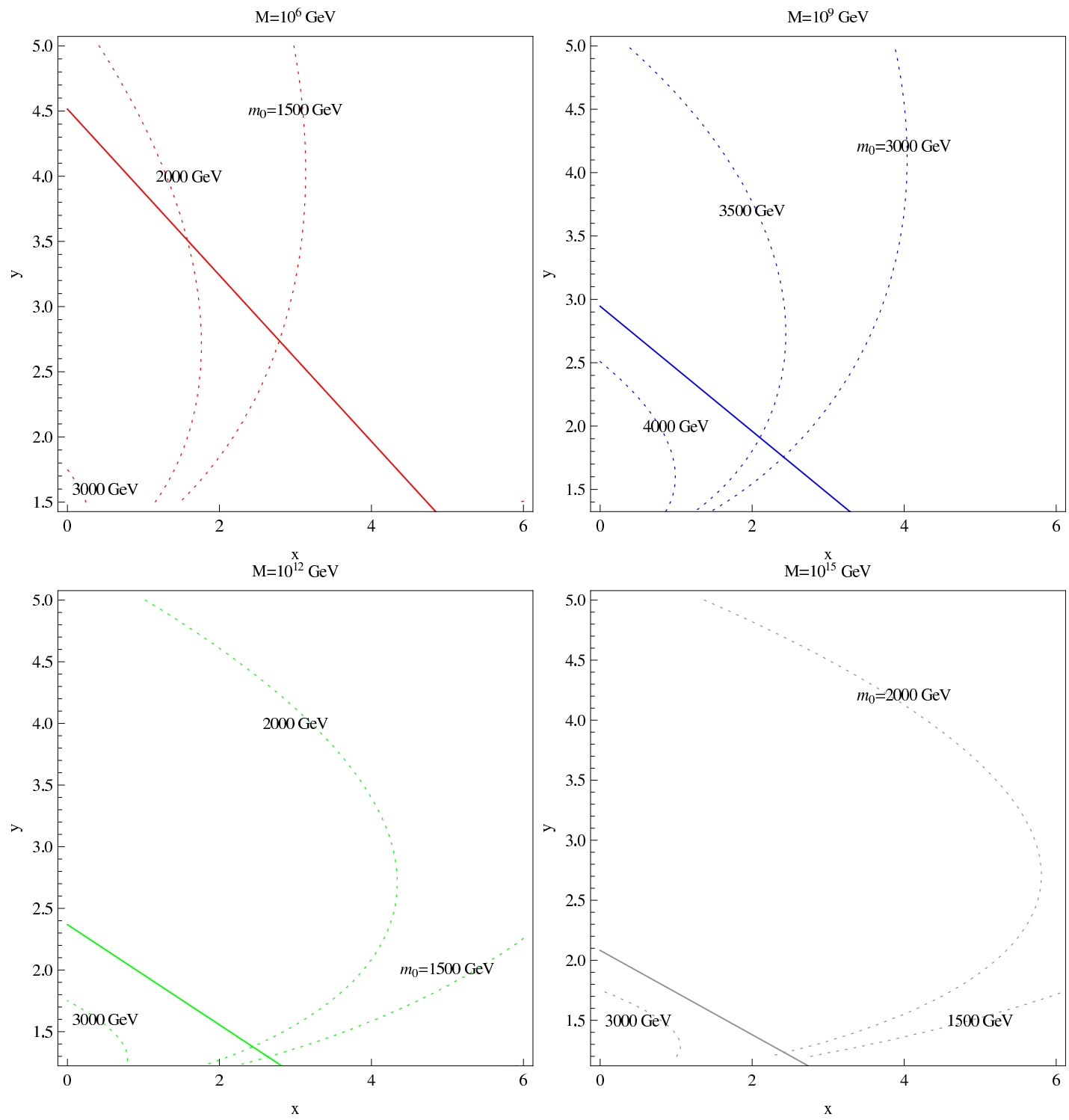

Figure 2. Contour of Higgs mass projected to the two-parameter plane of $(x, y)$ for $\tan \beta=20$ and $M=\left\{10^{6}, 10^{9}, 10^{12}, 10^{15}\right\} \mathrm{GeV}$. The focusing lines of figure 1 are shown simultaneously. In each panel, we show the sensitivity of Higgs mass to the input mass parameter $m_{0}$. It clearly shows that the observed Higgs mass constrains $m_{0}$ in the range $1 \mathrm{TeV} \leq m_{0} \leq 2.5 \mathrm{TeV}$. 

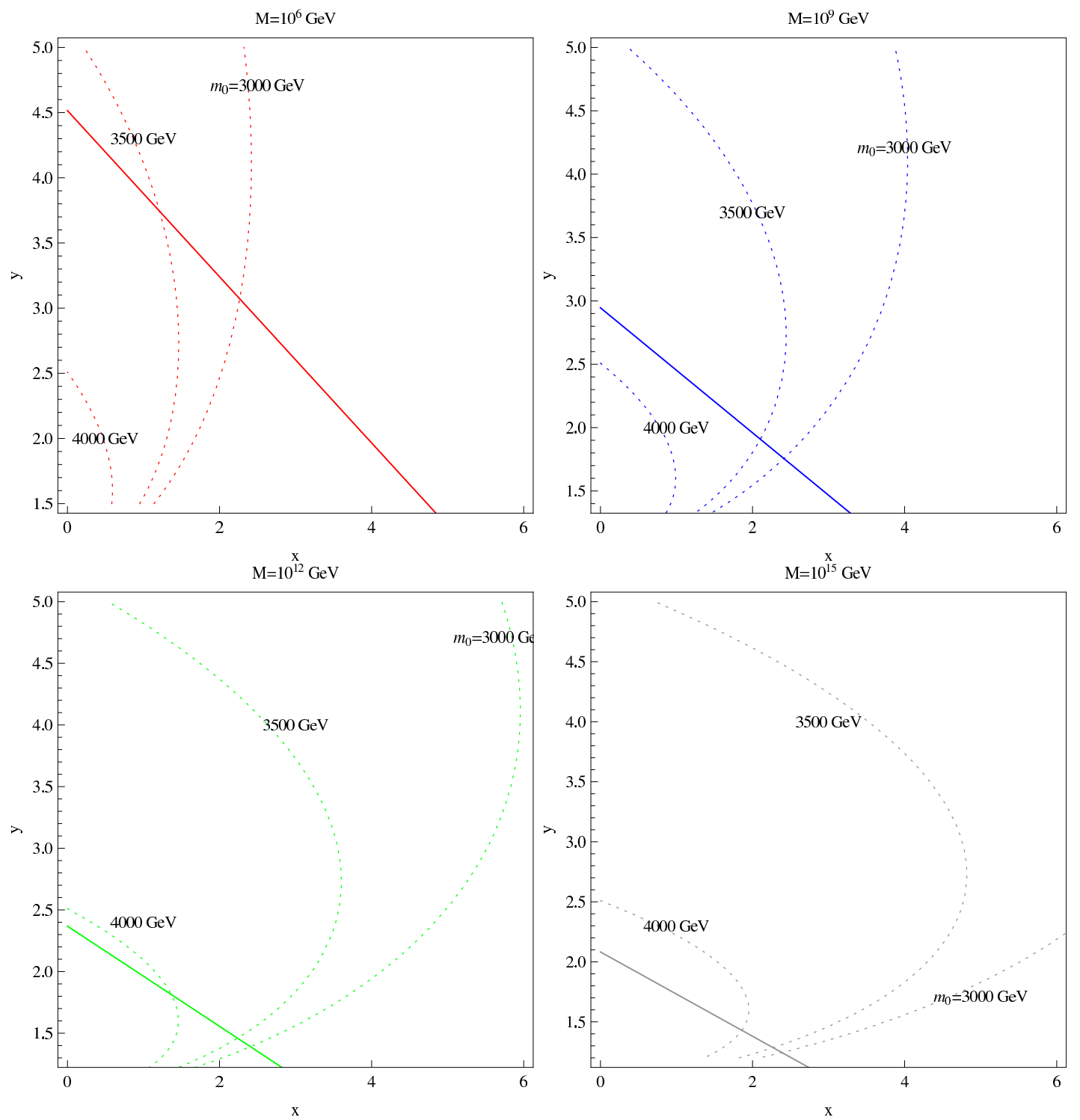

Figure 3. Same as figure 2 for $\tan \beta=5$. In this case there is about $6 \mathrm{GeV}$ reduction in the tree-level part of Higgs mass in compared with the previous choice $\tan \beta=20$ in figure 2, so larger loop contribution to the Higgs mass is required. 

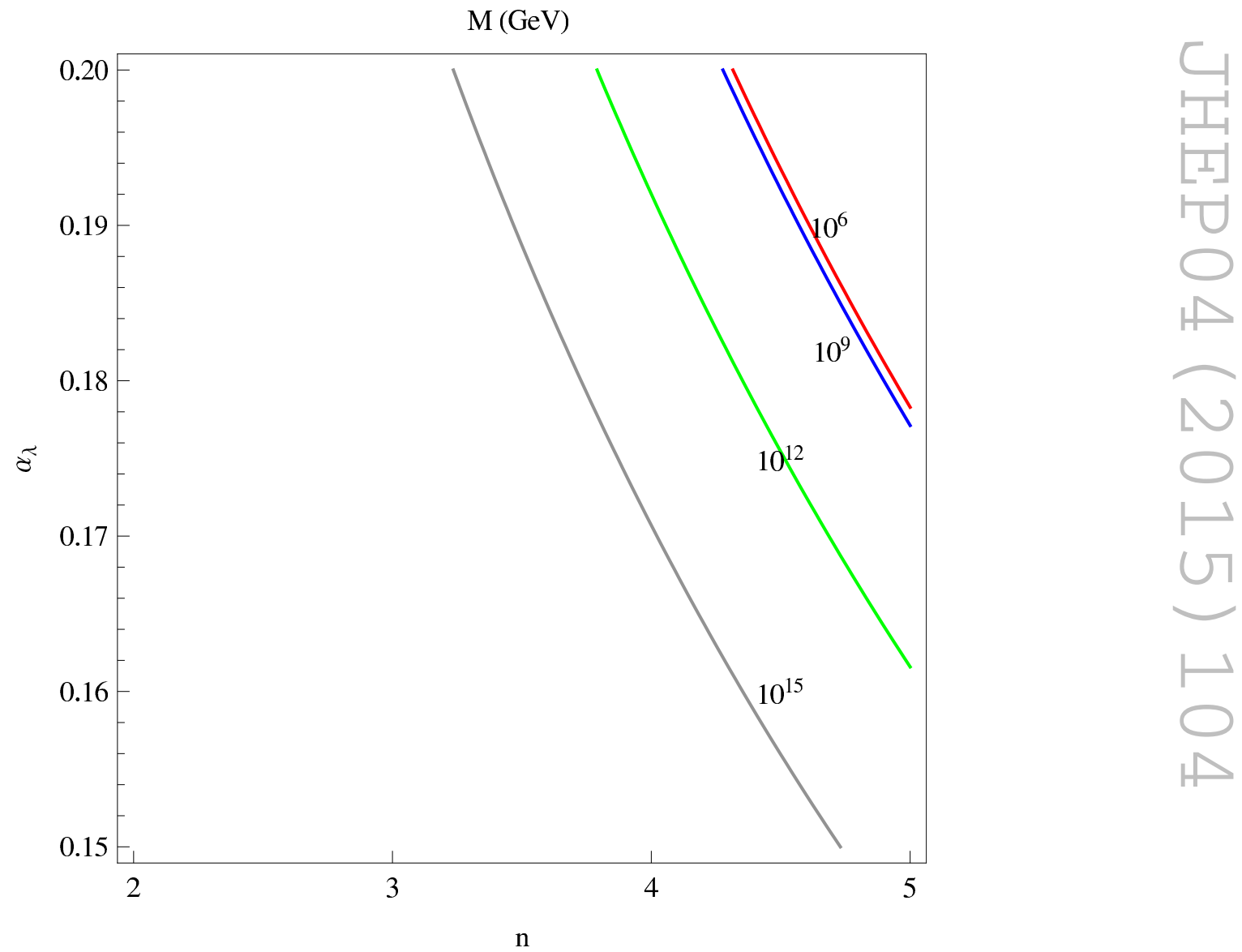

Figure 6. Solution to the focusing condition in eq. (2.7) projected to two-parameter plane of $\left(n, \alpha_{\lambda_{S}}\right)$ for $M=\left\{10^{6}, 10^{9}, 10^{12}, 10^{15}\right\} \mathrm{GeV}$. It has been verified that each solution satisfies the consistent conditions in eq. (2.8). 